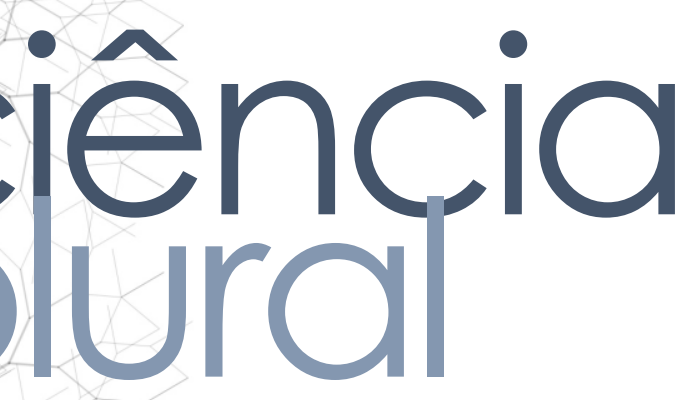

\title{
PREVALÊNCIA DE MALOCLUSÃO EM DENTIÇÃO DECÍDUA NOS ALUNOS DO ENSINO PRÉ- ESCOLAR DO CONCELHO DE PORTO DE MÓS (PORTUGAL)
}

Prevalence of malocclusion in deciduous dentition in preschool students in the municipality of Porto de Mós (Portugal)

Prevalencia de maloclusión en dentición temporal en preescolares del municipio de Porto de Mós (Portugal)

Irene Ventura • Professora de Odontopediatria do Instituto Universitário Egas Moniz-Portugal • E-mail: imvcr.ireneventura@gmail.com

José Miguel Jorge • MSc Instituto Universitário Egas Moniz-Portugal • E-mail: zemiguel.mj@gmail.com

Ana Raquel Barata • Professora do Odontopediatria do Instituto Universitário Egas Moniz-Portugal • E-mail: raquelgarciabarata@gmail.com

Gunel Kizi • Professora de Odontopediatria do Instituto Universitário Egas MonizPortugal • E-mail: gunelkizi@outlook.com

Autora correspondente:

Irene Ventura • E-mail: imvcr.ireneventura@gmail.com 


\section{RESUMO}

Introdução: A maloclusão é considerada a segunda doença oral mais comum em crianças e jovens adultos. É importante saber identificar estas alterações de forma a permitir um adequado desenvolvimento da oclusão. $O$ diagnóstico de um desenvolvimento anormal na dentição decídua é relevante para a prevenção e quando necessário uma intervenção precoce. Objetivo: O objetivo deste estudo transversal foi avaliar a prevalência de maloclusão em crianças com dentição decídua e relacionar com diferentes parâmetros. Metodologia: Observaram-se 300 crianças de ambos os sexos, com idades compreendidas entre os 3 e os 6 anos. A recolha de dados foi realizada a partir do exame clínico com kits de observação (espelho bucal básico descartável, sonda descartável, babador, luvas e sacos descartáveis), sob luz natural e na cadeira escolar, no Agrupamento de Escolas de Porto de Mós, Distrito Sanitário de Leiria. Analisaram-se as seguintes características: Tipo de arco de Baume, espaços primatas, diastemas, apinhamento, relação distal dos segundos molares decíduos, relação canina, sobremordida e sobressaliência, mordida anterior e posterior. Resultados: A prevalência de maloclusão registrada foi de 67,7\%, verificando-se ser mais baixa aos seis anos, sem apresentar diferenças significativas em ambos os sexos. A sobressaliência foi a maloclusão mais prevalente $(42,7 \%)$ nas crianças observadas, seguida da mordida aberta anterior (23,3\%). Registrou-se uma elevada prevalência de maloclusão nas crianças com arco de Baume tipo II, sem diastemas ou espaços primatas, com apinhamento, degrau distal, classe II canina ou desvio da linha média para a direita. Conclusões: Verificou-se existir uma elevada prevalência de maloclusão nas crianças do agrupamento de escolas do Concelho de Porto de Mós, estando relacionada com diferentes parâmetros oclusais.

Palavras-Chave: Crianças, Dentição decídua, Maloclusão.

\section{ABSTRACT}

Introduction: Malocclusion is considered a second most common oral disease in children and young adults. It is important to know how to identify these changes in order to allow proper development of the occlusion.The diagnosis of anabnormal development in primary dentition is relevant for prevention and when necessary, early intervention. Objective: The aim of this cross sectional study was to evaluate the prevalence of malocclusion in children with deciduous dentition and relate this prevalence with sex, age and with different occlusal parameters. Methodology: 300 children of both sexes, aged between 3 and 6 years were observed. Data collection was carried out from the clinical examination using observation kits, in schools, in the Group of schools of Porto de Mós, Health District of Leiria. The following characteristics were analyzed: Baume arc Type, spaces, diastemas, crowding, the deciduous second molars distal relation, canine relationship, Overbite and overjet, terior and posterior bite. Results: The recorded prevalence of malocclusion was $67.7 \%$, was lower at six years old, without any differences in both sexes. The overjet as the most prevalent malocclusion $(42.7 \%)$ in the observed children. There was a h prevalence of malocclusion in children with type II Baume arch, without spaces primate spaces, with distal step, canine class II or with the middle line shifted to right. Conclusions: There there was a high prevalence of malocclusion in 
children of the schools in the municipality of Porto de Mós, and is related to the different occlusal parameters.

Keywords: Children, Deciduous dentition, Malocclusion.

\section{RESUMEN}

Introducción: La maloclusión se considera la segunda enfermedad bucal más común en niños y adultos jóvenes. Es importante identificar estos cambios para llograr un adecuado desarrollo de la oclusión. El diagnóstico precoz del desarrollo anormal en la dentición temporal es relevante para la prevención y cuando sea necesario una intervención temprana. Objetivo: El objetivo de este estudio transversal fue evaluar la prevalencia de maloclusión en niños con dentición temporal y relacionarla con diferentes parámetros. Metodología: Fueran observados 300 niños de ambos sexos, con edades entre los 3 y los 6 años. La recogida de datos del examen clínico se realizó mediante kits de observación, en un entorno escolar, en el Grupo Escolar de Porto de Mós, Distrito Sanitario de Leiria. Se analizaron las siguientes características: tipo de arco de Baume, espacios primates, diastemas, apiñamientos, relación distal de los segundos molares deciduos, relación canina, sobremordida horizontal y vertical, mordida anterior y posterior. Resultados: La prevalencia de maloclusión registrada fue de $67,7 \%$, la cual resultó ser menor a los seis años, sin mostrar diferencias significativas en ambos sexos. El overjet fue la maloclusión más prevalente $(42,7 \%)$ en los niños observados, seguida de la mordida abierta anterior $(23,3 \%)$. Hubo una alta prevalencia de maloclusión en niños con arco de Baume tipo II, sin diastemas ni espacios de primates, con apiñamiento, paso distal, canino clase II o desviación de la línea media hacia la derecha. Conclusiones: Se encontró que hay una alta prevalencia de maloclusión en niños dela comunidad escolar del municipio de Porto de Mós, y que se relaciona, con diferentes parámetros oclusales.

Palabras clave: Niños, Dentición temporal, Maloclusión. 


\section{Introdução}

Segundo a Organização Mundial de Saúde (O.M.S.) a maloclusão é a segunda doença oral mais comum em crianças e jovens adultos depois da cárie dentária. É recomendado pela mesma, a elaboração de pesquisas periódicas dos principais problemas de saúde oral, sendo estes importantes na identificação de alterações oclusais durante o crescimento e na determinação da distribuição das condições de saúde oral na população $0^{1,2}$.

A oclusão na dentição decídua desempenha um papel importante na projeção da oclusão na dentição permanente, uma vez que o conjunto de características desta dentição estabelece as bases para uma erupção e alinhamento dentário adequados ${ }^{3-4}$.

Assim, o conhecimento dos fatores etiológicos e do panorama epidemiológico da maloclusão é um recurso útil para o planejamento de políticas de saúde pública e avaliação dos resultados dos tratamentos, auxiliando na tomada de decisões por parte dos médicos dentistas e das entidades públicas. O diagnóstico precoce pode facilitar o tratamento ortodôntico, preventivo ou interceptivo, aproveitando o potencial de crescimento da criança, evitando tratamentos mais complexos e honerosos 5 .

É essencial que o médico dentista compreenda e reconheça a amplitude das alterações que ocorrem normalmente na dentição, para ser capaz de diagnosticar ou interceptar qualquer desenvolvimento anormal. As condições que predispõem à maloclusão na dentição permanente, devem ser detectadas no início da dentição decídua de forma a que seja possível tanto a prevenção como uma intervenção precoce $^{6,7}$.

Este trabalho teve como objetivos, verificar a prevalência do tipo de maloclusão em crianças dos três aos seis anos de idade; registrar relacionando a idade, o sexo e diferentes parâmetros oclusais (tipo de arco de Baume, espaços primatas, diastemas, apinhamento, relação distal dos segundos molares decíduos, lação canina, sobremordida e sobressaliência, mordida anterior e posterior) com a valência da mesma. 


\section{Metodologia}

O estudo foi realizado nas escolas públicas do Concelho de Porto de Mós, após a aprovação do Diretor do Agrupamento de Escolas dessa localidade. Foi previamente submetido e aprovado por unanimidade pela Comissão de Ética da Egas Moniz, Cooperativa de Ensino Superior CRL. Foram selecionadas 300 crianças entre os 3 e 6 anos, sem alterações craniofaciais e cujos pais/tutores assinaram o consentimento informado.

A observação foi realizada apenas por um examinador sendo a calibração efetuada através da observação repetida, de 10 em 10 crianças, no dia posterior, de forma a validar o diagnóstico. Os dados foram registrados numa ficha de diagnóstico baseada no modelo da Organização Mundial de Saúde (O.M.S.), à qual foi atribuído um número de registro, preservando a identidade das crianças. Foram consideradas as seguintes variáveis: tipo de arco de Baume; espaços primatas; diastemas; apinhamento; relação distal dos segundos molares decíduos; relação canina; sobremordida aumentada; sobressaliência aumentada; desvio da linha média; mordida aberta anterior; mordida cruzada anterior; mordida cruzada posterior direita, esquerda e bilateral; mordida topo a topo posterior direita, esquerda e bilateral.

Realizou-se uma estatística descritiva para a amostra populacional final (frequências absolutas e relativas) e estatística inferencial. Utilizou-se o teste Binomial para comparação de duas proporções e o teste Qui Quadrado independente para a relação das variáveis quantitativas. $O$ teste de Fisher foi utilizado para análise estatística de variáveis de dois níveis $(\mathrm{gl}=1)$.

Os dados recolhidos foram analisados estatisticamente no programa informático SPSS (Statistical Package for the Social Sciences) versão 22.0 para o Windows, e resumidos em tabelas e gráficos no programa de software Microsoft ffice Excel 2007 SP3. Foram considerados diferenças estatisticamente significativas: ultados para $\mathrm{p}<0,05$. 


\section{Resultados}

As 300 crianças foram distribuídas equitativamente por ambos os gêneros, $50,0 \%(n=150)$ do gênero masculino e 50,0\% ( $n=150)$ do gênero feminino. No que diz respeito à distribuição da idade, verificou-se que as crianças com 3 anos representavam 27,0\% $(\mathrm{n}=85)$ do total da amostra, as crianças com 4 e 5 anos representavam igualmente $34,0 \%(n=100)$ e as com 6 anos 5,0\% (n=15).

\section{Estudo do espaço}

Verificou-se uma prevalência de crianças com arco de Baume tipo I de 67,7\% $(n=203)$. Observou-se um predomínio de meninos com arco de Baume tipo I [73,3\% $(n=110)$ versus $62,0 \%(n=93)]$ e de meninas com arco de Baume tipo II $[38,0 \%(n=57)$ versus $26,7 \%(n=40)]$, sendo esta diferença estatisticamente significativa $(p=0.048)$. Para o tipo de arco de Baume a prevalência nas diferentes idades não foisignificativa $(p=0,778)$.

A prevalência de crianças com espaços primatas na arcada superior foi de $87,3 \%$ ( $n=262)$, mais elevada nos meninos com espaços primatas na arcada superior, tendodiferençasignificativa $[92,0 \% \quad(n=138)$ versus $82,7 \% \quad(n=94), \quad(p=0,023)]$. A presença de espaços primatas na arcada superior nas diversas idades não apresentou diferenças $(p=0,248)$, sendo que na arcada inferior a prevalência dos espaços primatas foi de $70,7 \%(n=212)$, também sem diferenças significativas $(p=0,254)$ em ambos os sexos. Verificou-se um aumento de espaços primatas na arcada inferior, em crianças com 3 anos, com significância [78,8\% $(n=67),(p=0.003)]$. A percentagem de crianças com espaços primatas na arcada superior e inferior foi de 68,0\% (n=204), semdiferenças estatisticamente significativas em ambos os sexos $(p=0,063)$. Por outro lado, observou-se um número mais elevado de crianças com espaços primatas em ambas as arcadas aos 3 anos $(76,5 \% \quad(n=65)$ mostrando diferença significativa $(p=0,016)$.

A prevalência de crianças com diastemas na arcada superior foi de 70,3\% 211), não apresentando diferenças estatisticamente significativas em ambos os os $(p=0,312)$ nem nas diferentes idades $(p=0,503)$. Registrou-se um predomínio de nças com diastemas na arcada inferior de 67,3\% (n=202), não apresentando 
diferenças estatisticamente significativas em ambos os sexos $(p=0,065)$ nem nas diferentes idades $(p=0,148)$. A percentagem de crianças com diastemas em ambas as arcadas foi de 60,0\% (n=180). Observou-se uma prevalência mais elevada no gênero masculino, sendo esta diferença estatisticamente significativa $[67,3 \% \quad(n=101)$ versus52,7\% $(n=79),(p=0,013)]$, porém, nas diferentes idades não apresentou $\operatorname{significância}(p=0,310)$.

Em relação ao apinhamento dentário na arcada superior, observou-se uma percentagem de 9,3\% (n=28), quenão representa diferença estatisticamente significativa em ambos os sexos $(p=0,552)$ e nas diferentes idades $(p=0,790)$. A prevalência de apinhamento dentário na arcada inferior foi de 25,7\% (n=77), não apresentou significância em ambos os sexos $(p=0,428)$, assim como nas diferentes idades $(p=0,947)$. Registrou-se uma prevalência de apinhamento dentário em ambas as arcadas de 6,7\% $(n=20)$, sem diferenças estatísticas relevantes nos diferentes sexos $(p=1,000)$ e idades $(p=0,144)$.

Cerca de 52,7\% ( $n=158)$ das crianças apresentaram um plano terminal vertical e $41,7 \%(n=125)$ plano terminal mesial e 5,7\% $(n=17)$ plano terminal distal, não mostrandodiferenças estatisticamente significativas em ambos os sexos $(p=0,740)$. A presença de vários planos nas diversasidades não representou diferenças estatisticamente significativas $(p=0,729)$.

Registrou-se uma prevalência de crianças com classe I canina de 66,7\% $(n=200), 28,3 \%(n=85)$ apresentavam classe II e 5,0\% ( $n=15)$ classe III, não havendo significância estatísticaem ambos os sexos $(p=0,534)$ e nas diferentes idades $(p=0,097)$.

A percentagem de crianças com a linha média normal foi de 89,0\% ( $n=267)$, sendo que 7,0\% (n=21) apresentavam a linha média desviada para a direita e 4,0\% $(n=12)$ para a esquerda, semdiferenças significativas em ambos os sexos $(p=0,825)$. A prevalência de crianças com desvio da linha média nas diferentes idades não foi ignificativa $(p=0,845)$ 


\section{Parâmetros oclusais}

A sobremordida aumentada afetou cerca de 21,7\% (n=65) das crianças, não existindo diferenças em ambos os sexos $(p=0,674)$ e nas diferentes idades $(p=0,157)$.

Registrou-se uma prevalência de crianças com sobressaliência aumentada de $42,7 \%(n=128)$, sem existir diferenças em ambos os sexos $(p=0,907)$, porém foi mais elevada nas crianças com 4 anos, sendo esta diferença significativa [51,0\% ( $n=51)$, $(\mathrm{p}=0,011)]$.

A mordida aberta anterior afetou cerca de $23,3 \% \quad(n=70)$ das crianças, não existindo diferenças estatisticamente significativas em ambos os sexos $(p=1,000)$. Esta apresentou-se mais elevada nas crianças com 3 anos $[37,6 \%(n=32),(p=0,001)]$.

Registrou-se uma prevalência de crianças com mordida cruzada anterior de 10,0\% (n=30), não existindo diferenças estatisticamente significativas em ambos os sexos $(p=0,848)$ assim como nas diferentes idades $(p=0,775)$. A mordida cruzada posterior direita afectou $7,7 \%(n=23)$ das crianças, sem diferença significativa em ambos os sexos $(p=0,192)$ e nas diferentes idades $(p=0,682)$.

Cerca de 5,3\% ( $n=16)$ das crianças apresentaram mordida cruzada posterior esquerda, com “valor p" em ambos os sexos 0,198 e nas diferentes idades 0,341. Por outro lado registrou-se uma prevalência de crianças com mordida cruzada posterior bilateral de 1,7\% ( $n=5)$, com “valor $p$ " em ambos os sexos 0,371 assim como nas diferentes idades 0,516 . Tanto a mordida cruzada posterior esquerda como a bilateral não apresentaram diferença significativa.

A prevalência de crianças com mordida topo a topo posterior direita foi de 2,0\% ( $\mathrm{n}=6)$, com valor $\mathrm{p}$ em ambos os sexos 0,684e nas diferentes idades 0,395. Em relação a mordida topo a topo posterior esquerda foi de 3,3\% (n=10), com valor p em ambos os sexos 0,750 e nas diferentes idades 0,510. Por fim registrou-se uma prevalência de crianças com mordida topo a topo posterior bilateral de $1,0 \%(n=3)$, presentando valor de p em ambos os sexos 1,000 assim como nas diferentes idades 71. Em qualquer dos casos de mordida topo a topo anteriormente citados, não se servou diferenças significativas. 


\section{Prevalência da maloclusão}

Registrou-se uma prevalência de crianças com maloclusão de 67,7\% (n=203), não existindo diferenças em ambos os sexos $(p=0,805)$. Em relação à idade, verificouse uma prevalência mais baixa de maloclusão nas crianças com 6 anos (tabela 1), sendo esta diferença significativa $[26,7 \% \quad(n=4), \quad(p=0,004)]$. Observou-se uma prevalência mais elevada de maloclusão nas crianças com arco de Baume tipo II $[79,4 \%(n=77)$ versus $62,1 \%(n=126)]$, sendo esta diferença relevante $(p=0.001)$ (tabela 2).

Tabela 1 - Prevalência de maloclusão nas diferentes idades. Porto de Mós-Portugal, 2016.

\begin{tabular}{ccccccc}
\hline Idade & \multicolumn{2}{c}{ Sem maloclusão } & \multicolumn{2}{c}{ Com maloclusão } & \multicolumn{2}{c}{ Total } \\
\hline 3 anos & 22 & $25,9 \%$ & 63 & $74,1 \%$ & 85 & $100 \%$ \\
4 anos & 33 & $33,0 \%$ & 67 & $67,0 \%$ & 100 & $100 \%$ \\
5 anos & 31 & $31,0 \%$ & 69 & $69,0 \%$ & 100 & $100 \%$ \\
6 anos & 11 & $73,3 \%$ & 4 & $26,7 \%$ & 15 & $100 \%$ \\
Total & 97 & $32,3 \%$ & 203 & $67,7 \%$ & 300 & $100 \%$ \\
\hline
\end{tabular}

Tabela 2 - Maloclusão/ Arco de Baume. Porto de Mós-Portugal, 2016.

\begin{tabular}{lllllll}
\hline Arco de Baume & \multicolumn{2}{l}{ Sem maloclusão } & \multicolumn{2}{l}{ Com maloclusão } & Total \\
\hline Arco tipo I & 77 & $37,9 \%$ & 126 & $62,1 \%$ & 203 & $100 \%$ \\
Arco tipo II & 20 & $20,6 \%$ & 77 & $79,4 \%$ & 97 & $100 \%$ \\
Total & 97 & $32,3 \%$ & 203 & $67,7 \%$ & 300 & $100 \%$ \\
\hline
\end{tabular}

As crianças sem espaços primatas em ambas as arcadas apresentaram uma elevada prevalência de maloclusão [83,3\% ( $n=80)$ versus 60,3\% ( $n=123),(p=0.001)$ ] (tabela 3), assim como foi observada uma prevalência mais elevada de maloclusão as crianças sem diastemas em ambas as arcadas $[77,5 \%(n=93)$ versus $61,1 \%(n=110)$, $p=0.001)]$ (tabela 4). Foi observada uma diferença significativa em ambos. 
Tabela 3 - Maloclusão/Espaços primatas em ambas as arcadas Porto de MósPortugal, 2016.

\begin{tabular}{lllllll}
\hline Espaços Primatas & \multicolumn{2}{l}{ Sem maloclusão } & \multicolumn{2}{l}{ Com maloclusão } & \multicolumn{2}{l}{ Total } \\
\hline Sem espaços primatas & 16 & $16,7 \%$ & 80 & $83,3 \%$ & 96 & $100 \%$ \\
Com espaços primatas & 81 & $39,7 \%$ & 123 & $60,3 \%$ & 204 & $100 \%$ \\
Total & 97 & $32,3 \%$ & 203 & $67,7 \%$ & 300 & $100 \%$ \\
\hline
\end{tabular}

Tabela 4- Maloclusão/Diastemas em ambas as arcadas Porto de Mós-Portugal, 2016

\begin{tabular}{lcccccc}
\hline Diastemas & \multicolumn{2}{l}{ Sem maloclusão } & \multicolumn{2}{l}{ Com maloclusão } & \multicolumn{2}{l}{ Total } \\
\hline Sem diastemas & 27 & $22,5 \%$ & 93 & $77,5 \%$ & 120 & $100 \%$ \\
Com diastemas & 70 & $38,9 \%$ & 110 & $61,1 \%$ & 180 & $100 \%$ \\
Total & 97 & $32,3 \%$ & 203 & $67,7 \%$ & 300 & $100 \%$ \\
\hline
\end{tabular}

Registrou-se uma prevalência mais elevada de maloclusão nas crianças com apinhamento dentário, sendo esta diferença estatisticamente significativa [84,7\% $(n=72)$ versus15,3\% $(n=13),(p=0.001)]$ (tabela 5).

Tabela 5 - Maloclusão/ apinhamento. Porto de Mós-Portugal, 2016.

\begin{tabular}{cccccc}
\hline Apinhamento & \multicolumn{2}{c}{$\begin{array}{c}\text { Sem } \\
\text { maloclusão }\end{array}$} & \multicolumn{2}{c}{$\begin{array}{c}\text { Com } \\
\text { maloclusão }\end{array}$} \\
\hline Ausência & 84 & $39,1 \%$ & 131 & $60,9 \%$ & 96 \\
Presença & 13 & $15,3 \%$ & 72 & $84,7 \%$ & 204 \\
Total & 97 & $32,3 \%$ & 203 & $67,7 \%$ & 300 \\
\hline
\end{tabular}

Observou-se uma prevalência mais elevada de maloclusão nas crianças que resentavam o degrau distal como relação entre os segundos molares decíduos ,2\% ( $n=15),(p=0.001)]$, assim como uma prevalência mais elevada de maloclusão 
nas crianças com classe II canina [91,8\% $(n=78),(p=0.001)]$ o que mostrou relevância estatistica.

Em relação à prevalência de maloclusao nas crianças com a linha média desviada, esta foi mais prevalente para os desvios do mento para a direita, sendo esta relação estatisticamente significativa [95,2\% $(n=20),(p=0.008)]$ (tabela 9).

\section{Discussão}

Verificou-se que das 300 crianças observadas, 203 (67,7\%) apresentam maloclusão, não sendo verificadas diferenças significativas em ambos os gêneros $(p=0,805)$. Em estudos realizados em Portugal, com uma amostra da mesma idade as prevalências observadas variam. Ventura $(2005)^{8}$, num estudo realizado em Almada e Setúbal com 627 crianças registrou uma prevalência mais baixa $(44,0 \%)$, tal como Silva et al. (2009) ${ }^{9}$ num trabalho realizado em três escolas na freguesia de Alfena com 224 crianças (21,1\%), Costa (2011) 10 em 116 crianças de Alcoitão (48,3\%) e Gafaniz $(2015)^{11}$, num estudo com 100 crianças de um jardim de infância da Abrigada (53,0\%). Estes valores demonstram que a prevalência de maloclusão tem vindo a aumentar em Portugal.

Na Alemanha, Stahl e Grabowski (2003)12 observaram uma prevalência mais baixa de 42,0\% em 1225 crianças com dentição decídua e Wagner e HeinrichWeltzien $(2015)^{3}$, analisando apenas crianças com 3 anos observou uma prevalência semelhante de 45,2\%. Keski-Nisula et al. (2003) ${ }^{13}$ avaliando 489 crianças Finlandesas com idades entre os 4 e os 8 anos, registrou uma prevalência igual ao nosso estudo de 67,7\%. Na Lituânia, Kasparaviciene et al. (2014) ${ }^{14}$ observou 503 crianças com idades compreendidas entre os 5 e os 7 anos, registrando uma prevalência de 71,4\%.

Em estudos realizados no Brasil por Sousa, Pinto-Monteiro et al. (2014) ${ }^{15}$ com 732 crianças de Campinas e Normando et al. (2015) ${ }^{16}$ com 652 crianças da Amazónia, registraram prevalências igualmente elevadas de 62,4\% e 81,4\% respetivamente. Estes dados estão de acordo com as indicações da OMS, de que a maloclusão é uma ndição muito prevalente. 
Neste estudo registramos uma maior prevalência de maloclusão em crianças com 3 anos $(74,1 \%)$, seguido das crianças com 5 anos $(69,0 \%)$, das de 4 anos $(67,0 \%)$ e finalmente das crianças com 6 anos $(26,7 \%)$, sendo esta diferença estatisticamente significativa $(p=0,004)$. Estes resultados vão de encontro aos valores obtidos por Ventura (2005) 8 e Sousa, Pinto-Monteiro et al. (2014) ${ }^{15}$ onde observaram uma maior prevalência de maloclusão em crianças com 3 anos (54,0\% e 69,1\% respectivamente), diminuindo consideravelmente com o avançar da idade. Gafaniz (2015) ${ }^{11}$ observou uma maior prevalência em crianças com 4 anos (39,0\%) não encontrando relevância estatística.

Verificou-se que a prevalência de maloclusão na dentição decídua varia bastante entre as populações. Estas diferenças podem ser explicadas pelas características individuais, como fatores socioeconômicos, diferenças culturais e o tamanho das amostras estudadas.

\section{Conclusões}

De acordo com os objetivos propostos para este estudo, é permitido retirar diversas conclusões quanto à prevalência das maloclusões, e suas consequências para a dentição definitiva.

A prevalência de maloclusão registrada nesta amostra populacional foi de $67,7 \%$, o que permite concluir que a maloclusão é mais elevada para esta população quando comparada com outras regiões do país e do mundo, não se relacionando com o gênero, no entanto, aos 6 anos de idade observa-se uma diminuição estatisticamente significativa da maloclusão.

A sobressaliência aumentada e a mordida aberta anterior foram as maloclusões mais prevalentes neste estudo, a mordida aberta anterior tem maior prevalência aos 3 anos, enquanto a sobressaliência aumentada é mais elevada aos 4 nos. Ambas as maloclusões diminuíam com a idade, pelo que concluímos que uma a prevalência aos 3-4 anos pode estar associada a hábitos de sucção não nutritiva e da, que a diminuição da sua prevalência com a idade pode sugerir que a ninação destes hábitos, numa idade ideal pode permitir a sua autocorreção. 
Existe uma forte relação entre a maloclusão e a existência do degrau distal, apinhamento, classe II canina, linha média desviada para a direita, uso de chupeta, sucção digital, arco de Baume tipo II e a presença de cárie dentária, como têm vindo a ser demonstrado em vários estudos epidemiológicos, comprovando-se assim a nossa terceira hipótese de trabalho.

A análise do arco de Baume e dos espaços primatas sugerem uma maior tendência para o desenvolvimento de uma posterior maloclusão na dentição permanente, no sexo feminino. Por outro lado, ao avaliar os resultados do arco de Baume, espaços primatas, diastemas, apinhamento, plano terminal, relação canina e linha média podemos concluir que a grande maioria destas crianças tem tendência para um desenvolvimento dentário favorável bem como, maior propensão para se estabelecer uma relação classe I na dentição definitiva.

As mordidas cruzadas, quer anterior, quer posterior, têm baixa prevalência, não sendo observada nenhuma mordida em tesoura.

Entendemos ser necessário realizar mais estudos semelhantes em Portugal de forma a conhecer melhor a realidade do nosso país, no que diz respeito à prevalência de maloclusão e às consequências dos hábitos deletérios na dentição decídua, de forma a sensibilizar os profissionais de saúde, as autoridades sanitárias e a população, desenvolvendo políticas de saúde pública que apostem na prevenção, diagnóstico e interseção precoce.

\section{Referências}

1. Sharma A, Menon I, D.S. A, Dixit A. Prevalence of malocclusion and treatment needs among 12 to 15 years old school children in Muradnagar Uttar Pradesh. Journal of Dental and Medical Sciences. 2015; 14(1):60-65.

2. Sousa RV, Ribeiro GL, Firmino RT, Martins CC, Granville-Garcia AF, Paiva SM. Prevalence and associated factors for the development of anterior open bite and posterior crossbite in the primary dentition. Braz Dent J. 2014;25(4):336-342. 
3. Wagner Y, Heinrich-Weltzien R. Occlusal characteristics in 3-year-old children--results of a birth cohort study. BMC Oral Health. 2015; 15,94. doi:10.1186/s12903-015-0080-0.

4. Pinho T. A ortodontia intercetiva nas deformidades dento-maxilares. Nascer e Crescer - revista do hospital da criança maria pia. 2011;20(3):192-196.

5. Majorana A, Bardellini E, Amadori F, Conti G, Polimeni A. Timetable for oral prevention in childhood--developing dentition and oral habits: a current opinion. Prog Orthod. 2015; 16, 39. doi:10.1186/s40510-015-0107-8

6. Anitha XL, Asokan S. Occlusion characteristics of preschoolers in Chennai: a cross-sectional study. J Dent Child (Chic). 2013; 80(2): 62-66.

7. Lochib S, Indushekar KR, Saraf BG, SheoranN, Sardana D. Occlusal characteristics and prevalence of associated dental anomalies in the primary dentition. J Epidemiol Glob Health. 2015;5(2): 151-157. doi:10.1016/j.jegh.2014.07.001

8. Ventura I. Maloclusión en dentición temporal. Estudio epidemiológico en dos poblaciones del mismo distrito sanitario - Almada/Setúbal. Portugal[disertación]. Universidad de Sevilla, Sevilla; 2005.

9. Silva MJ, Ferreira AC, Silva CS, Teixeira ME, Valente CAP. O estado de saúde oral das crianças em idade pré-escolar e escolar de uma área urbana. Revista do hospital de crianças maria pia. 2009; XVIII(2).

10. Costa T. Influencia de parámetros infantiles y hábitos orales en la oclusión de niños de 3 a 5 años de edad [disertación]. Universidad de Granada, Granada; 2011.

11. Gafaniz, I. Prevalência de maloclusão em dentição decídua em crianças dos 3 aos 6 anos[dissertação]. Instituto Superior de Ciências da Saúde Egas Moniz, Monte da Caparica; 2015.

12. Stahl F, Grabowski R. Orthodontic findings in the deciduous and early mixed dentition--inferences for a preventive strategy. J Orofac Orthop. 2003; 64(6):401-416. doi:10.1007/s00056-003-0313-8

13. Keski-Nisula K, Lehto R, Lusa V, Keski-Nisula L, Varrela J. Occurrence of malocclusion and need of orthodontic treatment in early mixed dentition.Am J Orthod Dentofacial Orthop. 2003;124(6): 631-638. doi:10.1016/S0889540603006504 


\section{ciência
pural}

14. Kasparaviciene K, Sidlauskas A, Zasciurinskiene E, Vasiliauskas A, Juodzbalys G, Sidlauskas M, et al. The prevalence of malocclusion and oral habits among 5-7-year-old children.Med Sci Monit. 2014;20:2036-2042. doi:10.12659/MSM.890885

15. SousaRV, Pinto-Monteiro AK, Martins CC, Granville-Garcia AF, Paiva SM. Malocclusion and socioeconomic indicators in primary dentition. Braz Oral Res. 2014; 28:54-60.

16. Normando TS, Barroso RF, Normando D. Influence of the socioeconomic status on the prevalence of malocclusion in the primary dentition. Dental Press J Orthod. 2015;20(1): 74-78. doi:10.1590/2176-9451.20.1.074-078.oar 